Communication

\title{
Ionic Liquids as Carbene Catalyst Precursors in the One-Pot Four-Component Assembly of Oxo Triphenylhexanoates (OTHOs)
}

\author{
Anton Axelsson ${ }^{\dagger}$, Linda Ta ${ }^{\dagger}$ and Henrik Sundén * \\ Chemistry and Chemical Engineering, Chalmers University of Technology, Kemivägen 10, \\ 41296 Gothenburg, Sweden; E-Mails: antax@chalmers.se (A.A.); lindat@chalmers.se (L.T.) \\ $\dagger$ These authors contributed equally to this work. \\ * Author to whom correspondence should be addressed; E-Mail: sundenh@chalmers.se; \\ Tel.: +46-708-924671.
}

Academic Editor: Jason P. Hallett

Received: 24 October 2015 / Accepted: 18 November 2015 / Published: 27 November 2015

\begin{abstract}
Ionic liquids (ILs) are a convenient and inexpensive source of N-heterocyclic carbenes (NHCs). In this study, dialkyl imidazolium-based ILs are used as carbene precursors in a four-component synthesis of oxo triphenylhexanoates (OTHOs), where it was found that IL outperformed commonly used NHC precatalysts in terms of reaction efficiency. The reaction is highly stereoselective, delivering the anti-diastereomer (20:1 dr), and the OTHOs can be obtained in high-to-excellent yields. By virtue of the four-component reaction-setup, facile construction of the OTHO scaffold with a diverse set of functional groups ( 21 examples) can be achieved. In the context of sustainability, the IL can be recovered and reused several times without affecting selectivity or yield. Moreover, most compounds can be isolated by precipitation and filtration, mitigating the use of solvent-demanding chromatography.
\end{abstract}

Keywords: ionic liquid; NHC; OTHO; multicomponent reaction; carbene; organocatalysis; organogel; MCR; 1,6-ketoester 


\section{Introduction}

The separation, recovery, and reuse of a catalyst are major obstacles within homogeneous catalysis. In this respect, ionic liquids (ILs) have been shown to be an ideal medium for covalent and non-covalent catalyst immobilization and regeneration, due to their low vapor pressure [1-6]. Moreover, ILs themselves can also be used as catalysts [7,8]. This has been exemplified in several syntheses of heterocycles [9], cycloadditions [10], and acetylations of alcohols [11,12]. Rate enhancements seen in these reactions have been attributed to a range of activation modes such as hydrogen bonding [10,13], and Lewis acid [11] and base [12] catalysis. Other activation strategies can be invoked by deprotonation of dialkyl imidazolium-based ILs with a base, thus generating an $N$-heterocyclic carbene (NHC) (Figure 1a). In synthesis, NHCs derived from solid thiazolium, imidazolium and triazolium salts (Figure 1b) have proven to be efficient metal-free catalysts for a vast number of chemical transformations [14-21]. Although dialkyl imidazolium salts constitute the basis for a large number of commercial ILs, the use of such as NHC precatalysts in synthesis is underutilized compared to their solid counterpart [22-35].

a)

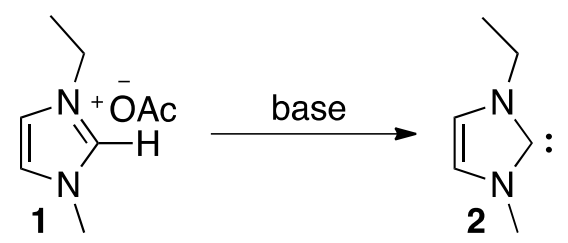

EMIMAC b)

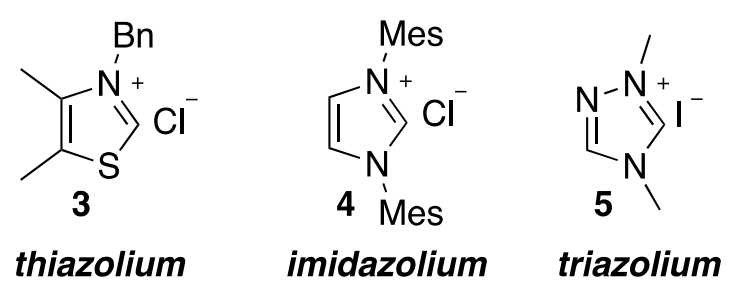

Figure 1. (a) Deprotonation of EMIMAc yields the corresponding NHC; (b) Common NHC precatalysts.

Solvents account for the majority of waste in chemical industry. For example, in fine chemical production, solvents comprise more than $80 \%$ of the mass utilization, consume about $60 \%$ of the overall energy used, and are responsible for $50 \%$ of the post-treatment greenhouse gas emissions [36-38]. A novel strategy to reduce solvent usage is to employ multicomponent reactions (MCRs) rather than multistep synthesis, hence omitting the use of several solvent-intensive purification steps [39]. Consequently, an MCR approach is less laborious and results in less waste per bond-forming step. Aside from the substantial sustainability benefits, MCRs enable the construction of complex molecules from simple starting materials in a single step.

Recently, we reported the use of EMIMAc as a precatalyst in the formal Michael addition of $\alpha, \beta$-unsaturated aldehydes to chalcones in the synthesis of oxo triphenylhexanoates (OTHOs) [40,41]. In this study it was also shown that one of the OTHOs was a potent low-molecular-weight gelator in hydrocarbon-based solvents. In order to further evaluate the OTHOs as gelators, a rapid and straightforward method of obtaining a library of compounds was required. For these purposes a multicomponent reaction would be ideal. Retrosynthetically, only three disconnections of the OTHO scaffold were required to enable the use of inexpensive and commercially available acetophenones, benzaldehydes, unsaturated aldehydes, and alcohols (Scheme 1) as starting materials for a multicomponent OTHO assembly. However, multiple challenges associated with side reactions such as benzaldehyde dimerization (benzoin 
condensation), $\alpha, \beta$-unsaturated aldehyde cross-reactivity, and accumulation of water in the reaction mixtures were expected with the MCR strategy.

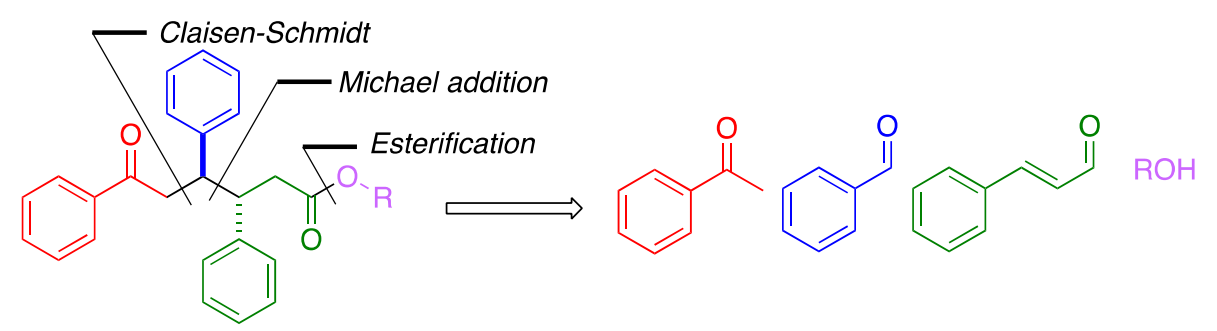

Scheme 1. Retrosynthesis of OTHOs.

\section{Results and Discussion}

The reaction between acetophenone, benzaldehyde, cinnamaldehyde, and methanol was chosen as a model reaction to investigate multicomponent OTHO synthesis. Initial experiments highlighted that sequencing the addition of reagents in an appropriate order could circumvent many of the expected problems (see Table 1 for a condensed optimization). A base screen indicated that strong bases such as tBuOK and NaOMe (Table 1, entry 1 and 2) were favored in the initial Claisen-Schmidt reaction and gave 10, however, in low yields. The ${ }^{1} \mathrm{H}$ NMR investigations displayed close to quantitative formation of the chalcone, indicating that the losses occur in the later steps of the MCR. Medium-strong bases such as TBD and $\mathrm{Cs}_{2} \mathrm{CO}_{3}$ (Table 1, entry 3 and 6) proved to be better suited and gave the product in moderate-to-good yields, but with elongated reaction times (up to $72 \mathrm{~h}$ ). The previous base of choice, DBU, failed to initiate the reaction (Table 1, entry 8). A range of trials with reduced amounts of solvents were performed in an attempt to speed up the reaction with the medium-strong bases. Gratifyingly, the strategy proved effective and full conversion could be achieved within $5 \mathrm{~h}$ when using the alcohol component of the reaction (in this case methanol) as the sole solvent in the first part of the MCR. Under these conditions TBD proved the most effective, yielding 10 in 66\% yield (Table 1, entry 4).

Regarding solvent selection, dichloromethane and acetonitrile proved the most suitable, whereas 1,4-dioxane and methanol were inferior in terms of yield (Table 1, entry 4, 5, 9, and 10). Acetonitrile was selected as the solvent of choice, given that it is considered to be more environmentally benign than dichloromethane. Three other ILs proved successful, namely BMIMCl, EMIMCl, and EMIMLactate, giving the product in 52\%-59\% yields; however, EMIMAc remained the IL of choice (Table 1, entry 11-13). These results indicate that the anion has a noticeable effect on the reaction outcome. A plausible explanation could be that the basicity of the anion is important, either by deprotonation of the NHC precursor [42] or by hindering protonation of the homoenolate [43], which would result in less side product formation. Notably, other commonly used non-IL precatalysts failed to promote the reaction (Table 1, entry 14-16). Moreover, in the case of EMIMLactate, the chiral anion did not induce any asymmetry in the reaction under our conditions. 
Table 1. Optimization of the IL-mediated four-component reaction of OTHOs ${ }^{\mathrm{a}}$.

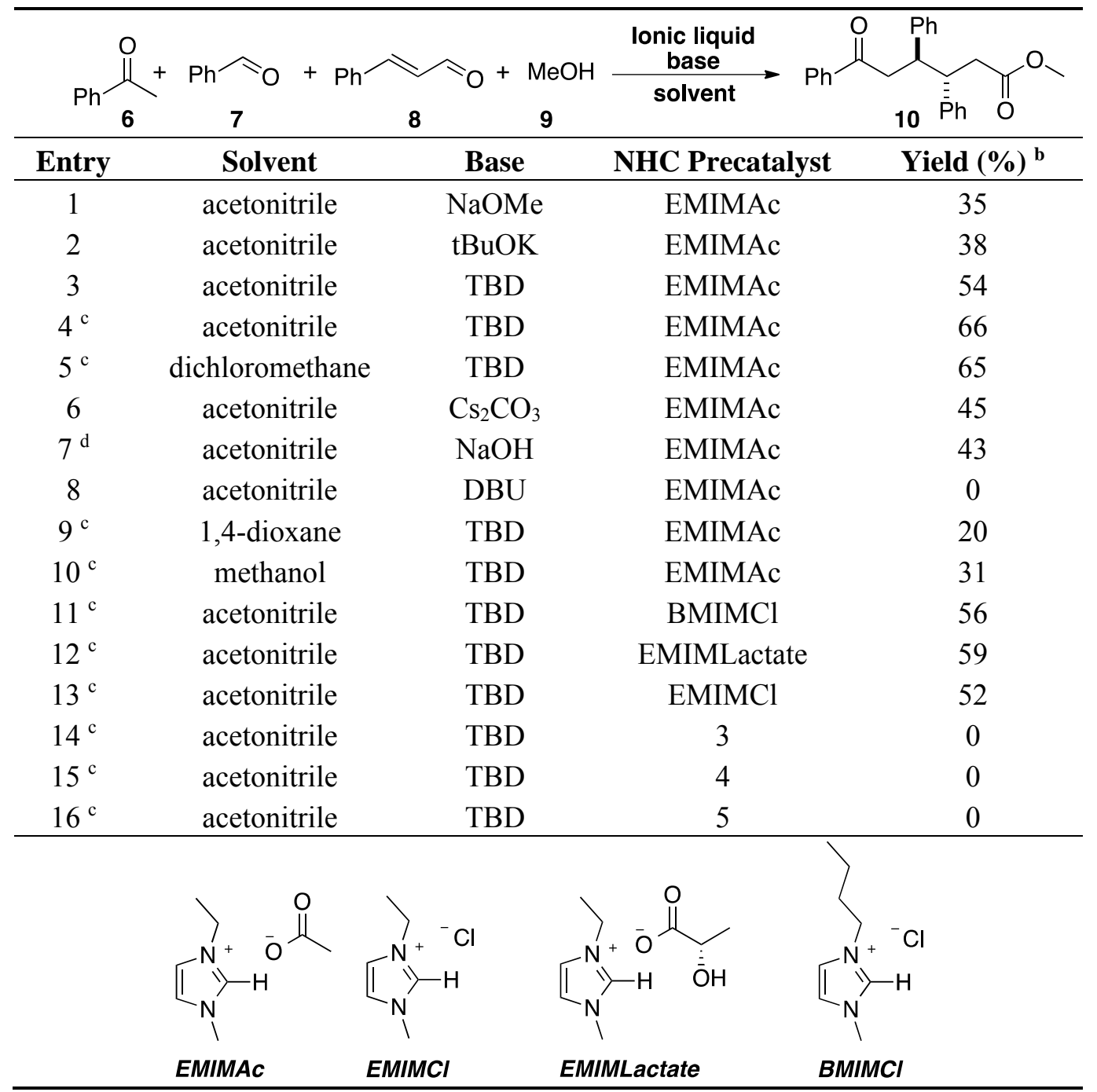

a. acetophenone ( $0.25 \mathrm{mmol})$, benzaldehyde (1.1 eq.), base (0.5 eq.), methanol (10 eq.), cinnamaldehyde (3 eq.), NHC precatalyst (2.5 eq.), solvent $(3 \mathrm{~mL})$ at room temperature; ${ }^{\text {b}}$ : Yields determined by ${ }^{1} \mathrm{H}$ NMR using 1,2,4,5-tetramethylbenzene as internal standard, $20: 1 \mathrm{dr} ;{ }^{\mathrm{c}}$ : Reduced amount of solvent; ${ }^{\mathrm{d}}$ : Reduced amount of solvent, 10 eq. of $\mathrm{NaOH}$ used.

Next, the scope of the reaction was systematically investigated in terms of the individual components (acetophenone, benzaldehyde, $\alpha, \beta$-unsaturated aldehyde, and alcohol) and the results are presented in Schemes 2 and 3. It was shown that the reaction is general and the acetophenone component could be varied to a high extent. For example, 1-(thiophen-2-yl)ethan-1-one performed well and the corresponding ketoester 13 was isolated in 83\% yield (Scheme 2). Halogen-substituted OTHOs, which are potentially important handles for traceless cross-coupling modifications, could be swiftly accessed in high yield (Scheme 2, entry 11 and 12). Both electron-rich and electron-deficient benzaldehydes can beneficially be used in the four-component assembly, providing compounds 16-22 in high yields (Scheme 2). Heteroaromatic components such as furfural are also tolerated and compound $\mathbf{2 3}$ can be isolated in $52 \%$ yield. Interestingly, it was found that cinnamaldehyde could replace benzaldehyde generating ketoester 24 in 55\% yield, resulting in the unprecedented introduction of a double bond to the OTHO scaffold. Furthermore, the $\alpha, \beta$-unsaturated aldehyde can also be altered with aromatic electron-donating and electron-withdrawing substituents (Scheme 3, entries 25-28). For example, 4-fluoro-cinnamaldehyde 
Catalysts 2015, 5

2056

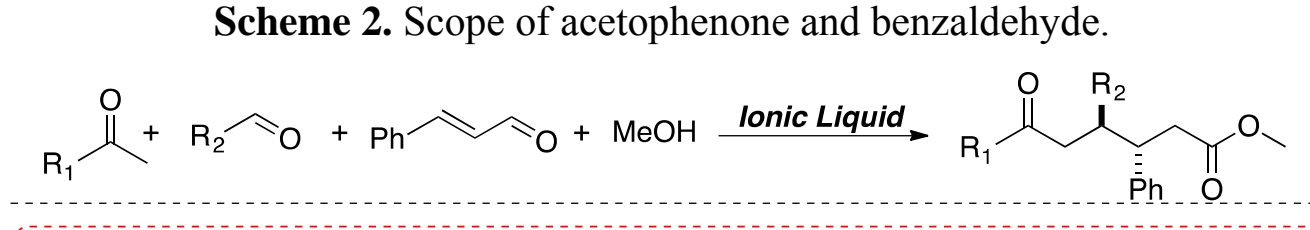

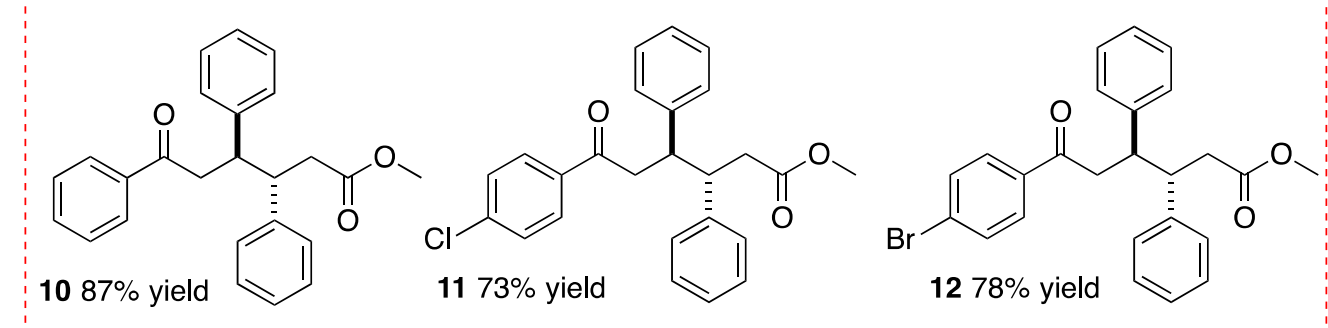

$$
\begin{aligned}
& \text { at } 0^{\circ} \text {. }
\end{aligned}
$$

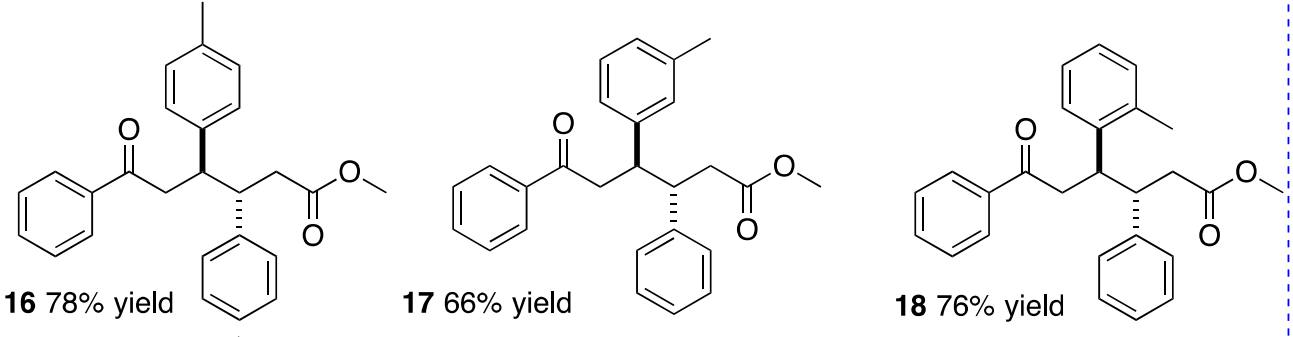

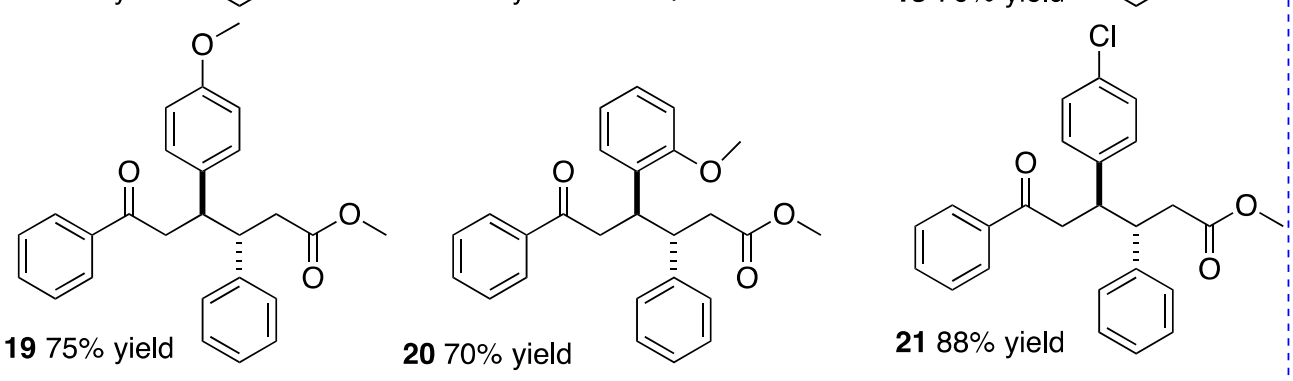

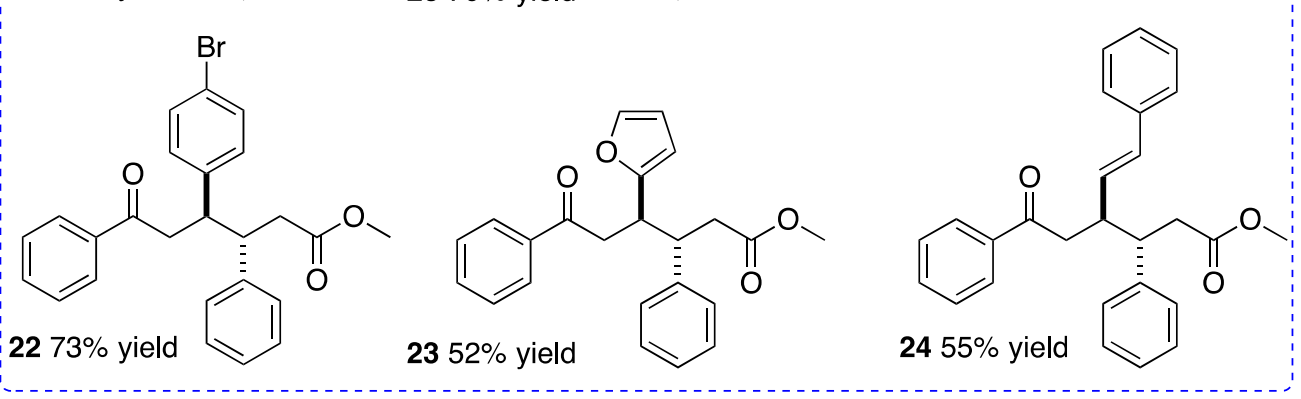


Scheme 3. Scope of unsaturated aldehyde and alcohol.

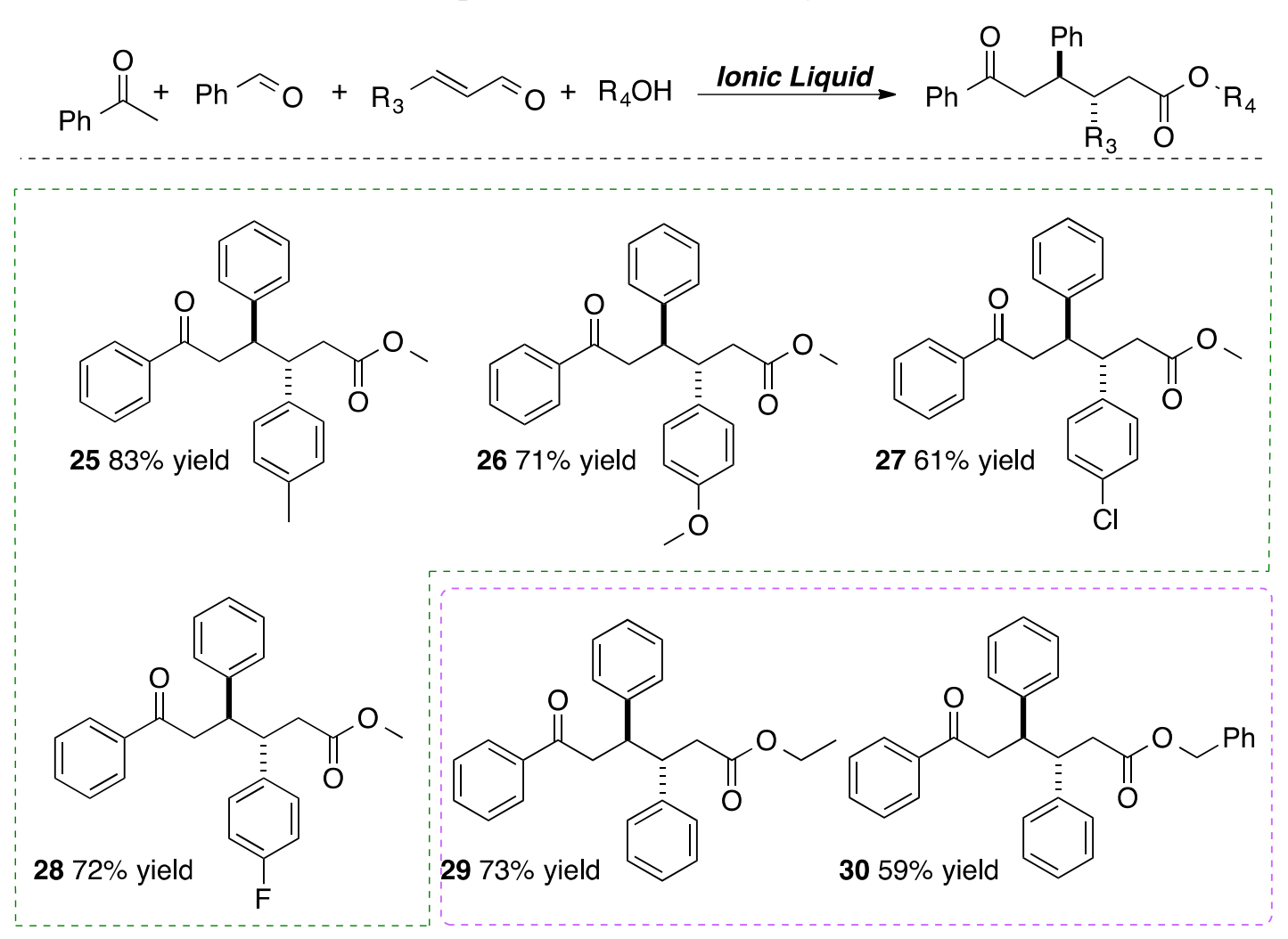

Significant efforts were made to recycle the IL by utilizing its low vapor pressure to further improve the sustainability of the reaction. Initial investigations showed that the original workup procedure was inappropriate with total deactivation of the IL occurring after two runs (Figure 2). The issue was identified as the usage of a methanol/water wash in the purification. When water is present the EMIMAc mixture needed to be dried at a higher temperature for a longer period of time, and this was suspected to be detrimental for the activity. By simply removing the water from the washing step, it was possible to recover and reuse the EMIMAc five consecutive times without reduced activity or selectivity. Efforts were made to elucidate the deactivation mechanism in the initial attempt. When mixtures of EMIMAc/water, EMIMAc/water/TBD, and pure EMIMAc were subjected to these conditions, no degradation or deactivation could be detected: hence, another deactivation route must be active. The ${ }^{1} \mathrm{H}$ and ${ }^{13} \mathrm{C}$ NMR investigations of the deactivated EMIMAc mixture showed the presence of several imidazolium species. Imidazolium-based ILs are known to degrade via several pathways, including dealkylation, hydrolysis, and rearrangements, all of which are highly influenced by other reaction additives $[44,45]$. Although the deactivation pathways remain elusive, it can be circumvented by using the pure methanol workup. 


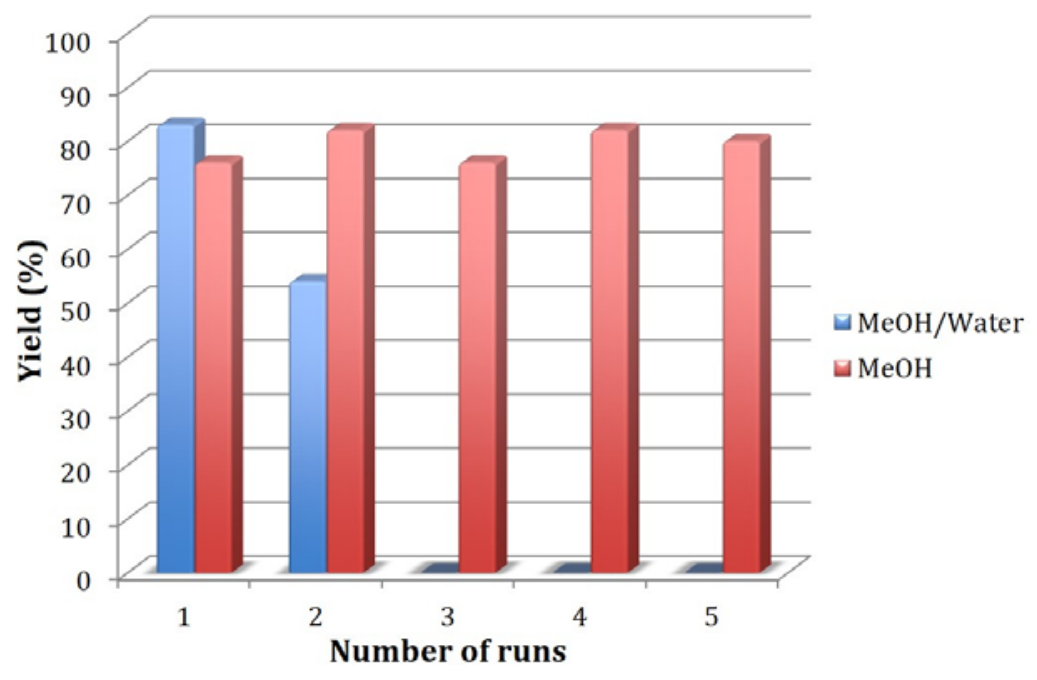

Figure 2. The results of the recycling experiments.

Mechanistically, deprotonation of EMIMAc yields the catalytically active carbene I which adds reversibly to cinnamaldehyde, forming Breslow intermediate II (Scheme 4). The Breslow intermediate then undergoes a formal Michael addition to the chalcone, formed in a base-catalyzed Claisen-Schmidt condensation between benzaldehyde and acetophenone, to give acyl azolium III. A nucleophilic attack by methanol delivers product $\mathbf{1 0}$ and regenerates the active carbene.

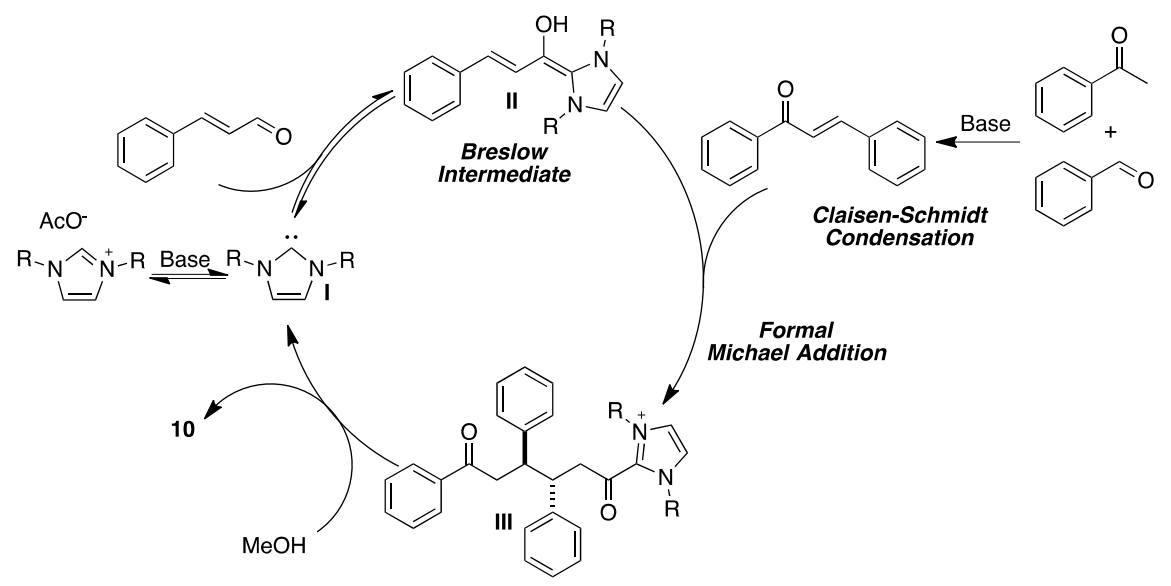

Scheme 4. The proposed mechanism for the four-component synthesis of OTHOs, R= Me, Et.

\section{Experimental Section}

\subsection{General Information}

Column chromatography was performed on automated column chromatography Biotage Isolera ${ }^{\mathrm{TM}}$ Spektra One with Biotage SNAP ${ }^{\circledR}-10 g$ KP-sil columns. Thin layer chromatography (TLC) was performed on Merck TLC plates pre-coated with silica gel $60 \mathrm{~F}_{254}$ (Art 5715, $0.25 \mathrm{~mm}$ ) and visualized with UV light (254 nm). The ${ }^{1} \mathrm{H}$ NMR (400 MHz), ${ }^{13} \mathrm{C}$ NMR (101 MHz) and ${ }^{19} \mathrm{~F}$ NMR (376 MHz) were recorded on a Varian 400. The chemical shifts are reported in parts per million $\delta(\mathrm{ppm})$ relative to the residual solvent peak $\mathrm{CDCl}_{3} ;{ }^{1} \mathrm{H} \mathrm{NMR}$ at $\delta 7.26 \mathrm{ppm}$ and ${ }^{13} \mathrm{C} \mathrm{NMR}$ at $\delta 77.2 \mathrm{ppm}$. Coupling constants $(J)$ are reported in Hertz $(\mathrm{Hz})$ and multiplicities are indicated by: s (singlet), dd (doublet of doublets), 
$\mathrm{t}$ (triplet), q (quartet), $\mathrm{m}$ (multiplet). Infrared (IR) spectra were recorded on a Perkin Elmer series FT-IR Spectrometer and are reported in wavenumber $\left(\mathrm{cm}^{-1}\right)$ as follows: vs (very strong), s (strong), $\mathrm{m}$ (medium), w (weak), br (broad). Melting points were recorded on a Büchi Melting Point B-545. The 1-Ethyl-3-methylimidazolium acetate (EMIMAc) was purchased from Sigma-Aldrich (Sweden AB, Stockholm, Sweden), produced by BASF $\geq 95 \%$ and dried in vacuo with heating prior to use. All other solvents and reagents were purchased from commercial sources and used without further treatments. NMR Spectras can be viewed in the supporting material (Figure S1-S45).

\subsection{Representative Procedure for the One-Pot Four-Component Synthesis of OTHOs}

To a $10 \mathrm{~mL}$ round bottom flask equipped with a magnet was added: 1,5,7-triazabicyclo[4.4.0]dec-5-ene (45.9 mg, $0.33 \mathrm{mmol})$, methanol $(211 \mathrm{mg}, 6.6 \mathrm{mmol})$, benzaldehyde $(77.8 \mathrm{mg}, 0.73 \mathrm{mmol})$, and acetophenone $(79.7 \mathrm{mg}, 0.66 \mathrm{mmol})$. The mixture was stirred at room temperature. If a precipitation was formed, a small amount of acetonitrile was added to dissolve the chalcone. When ${ }^{1} \mathrm{H}$ NMR indicated full consumption of acetophenone, a mixture of 1-ethyl-3-methylimidazolium acetate $(282.2 \mathrm{mg}, 1.7 \mathrm{mmol})$ and cinnamaldehyde $(262.4 \mathrm{mg}, 2.0 \mathrm{mmol})$ in acetonitrile $(7 \mathrm{~mL})$ was added. The mixture was stirred at room temperature. After $3 \mathrm{~h},{ }^{1} \mathrm{H}$ NMR indicated full consumption of chalcone and the volatiles were removed under reduced pressure. To the solids a methanol/water $(3: 1,7 \mathrm{~mL})$ mixture was added and the mixture was stirred violently for $30 \mathrm{~min}$. The heterogeneous mixture was filtered on a frit and washed first with water, then cold methanol/water (3:1) mixture, and lastly cold methanol. The solids were dried and the product was obtained as white powder $(214.0 \mathrm{mg}, 0.57 \mathrm{mmol}, 87 \%$ yield $)$.

\subsection{Procedure for Recycling of EMIMAc}

To a $25 \mathrm{~mL}$ round bottom flask equipped with a magnet was added: 1,5,7-triazabicyclo[4.4.0]dec-5-ene (48.7 mg, $0.35 \mathrm{mmol})$, methanol (223.1 mg, $7.0 \mathrm{mmol})$, benzaldehyde (81.7 $\mathrm{mg}, 0.77 \mathrm{mmol})$, and acetophenone $(84.7 \mathrm{mg}, 0.70 \mathrm{mmol})$. The mixture was stirred at room temperature. After $2 \mathrm{~h}$, cinnamaldehyde $(277.4 \mathrm{mg}, 2.1 \mathrm{mmol})$, acetonitrile $(7 \mathrm{~mL})$, and 1-ethyl-3-methylimidazolium acetate (297.9 $\mathrm{mg}, 1.8 \mathrm{mmol}$ ) were added to the stirring mixture. The mixture was stirred at room temperature. After $3 \mathrm{~h},{ }^{1} \mathrm{H}$ NMR indicated full consumption of chalcone and the volatiles were removed under reduced pressure. Methanol $(7 \mathrm{~mL})$ was added to the remaining solids and the mixture was stirred violently for $30 \mathrm{~min}$. The heterogeneous mixture was filtered on a frit and washed with cold methanol. The solids were dried and the product was obtained as white powder $(198.7 \mathrm{mg}, 0.53 \mathrm{mmol}, 76 \%$ yield). The methanolic mixture obtained from the filtration was collected and the volatiles were removed under reduced pressure, yielding an orange oil. The oil was re-used without further purification. The procedure was repeated for a total of five times.

Methyl-6-oxo-3,4,6-triphenylhexanoate (10) [40]: Synthesized from benzaldehyde, acetophenone, cinnamaldehyde and methanol according to the representative procedure. Obtained as a white solid (214.0 mg, $0.57 \mathrm{mmol}, 87 \%$ yield, 20:1 dr). ${ }^{1} \mathrm{H}$ NMR (400 MHz, $\left.\mathrm{CDCl}_{3}\right) \delta$ 7.66-7.61 (m, 2H), 7.47-7.41 (m, 1H), 7.36-7.26 (m, 10H), 7.25-7.15 (m, 2H), 3.65-3.56 (m, 1H), 3.48-3.40 (m, 1H) 3.37 (s, 3H), 3.28 (dd, $J=16.6,10.2 \mathrm{~Hz}, 1 \mathrm{H}), 2.90-2.87(\mathrm{~m}, 1 \mathrm{H}), 2.52-2.37$ (m, 2H). ${ }^{13} \mathrm{C}$ NMR $(101 \mathrm{MHz}$, 
$\left.\mathrm{CDCl}_{3}\right) \delta 198.7,172.7,142.5,142.3,137.2,132.9,128.9,128.8128 .5,128.4,128.0,127.3,127.0,51.5$, 48.0, 47.0, 43.9, 40.0. mp: $161.3-164.4^{\circ} \mathrm{C}$.

Methyl-6-(4-chlorophenyl)-6-oxo-3,4-diphenylhexanoate (11) [40]: Synthesized from benzaldehyde, 4-chloroacetophenone, cinnamaldehyde and methanol according to the representative procedure. Obtained as a white solid (225.3 mg, $0.55 \mathrm{mmol}, 78 \%$ yield, 20:1 dr). $\left.{ }^{1} \mathrm{H} \mathrm{NMR} \mathrm{(400} \mathrm{MHz}, \mathrm{CDCl}_{3}\right)$ d 7.58-7.51 (m, 2H), 7.39-7.26 (m, 10H), 7.26-7.15 (m, 2H), 3.61-3.53 (m, 1H), 3.47-3.39 (m, 1H), 3.37 (s, 3H), $3.21(\mathrm{dd}, J=16.5,10.1 \mathrm{~Hz}, 1 \mathrm{H}), 2.87(\mathrm{dd}, J=16.5,3.4 \mathrm{~Hz}, 1 \mathrm{H}), 2.52-2.37(\mathrm{~m}, 2 \mathrm{H})$. ${ }^{13} \mathrm{C} \mathrm{NMR}\left(101 \mathrm{MHz}, \mathrm{CDCl}_{3}\right) \delta 197.6,172.6,142.2,139.3,135.5,129.4,128.9,128.8,128.8,128.4$, $128.3,127.3,127.1,51.5,48.0,47.1,43.9,40.0 . \mathrm{mp}: 165.0-166.0^{\circ} \mathrm{C}$.

Methyl-6-(4-bromophenyl)-6-oxo-3,4-diphenylhexanoate (12) [40]: Synthesized from benzaldehyde, 4-bromoacetophenone, cinnamaldehyde and methanol according to the representative procedure. Obtained as a white solid (247.6 mg, $0.55 \mathrm{mmol}, 73 \%$ yield, 20:1 dr). ${ }^{1} \mathrm{H}$ NMR (400 $\left.\mathrm{MHz}, \mathrm{CDCl}_{3}\right)$ d 7.58-7.51 (m, 2H), 7.39-7.26 (m, 10H), 7.26-7.15 (m, 2H), 3.61-3.53 (m, 1H), 3.47-3.39 (m, 1H), $3.37(\mathrm{~s}, 3 \mathrm{H}), 3.21(\mathrm{dd}, J=16.5,10.1 \mathrm{~Hz}, 1 \mathrm{H}), 2.87(\mathrm{dd}, J=16.5,3.4 \mathrm{~Hz}, 1 \mathrm{H}), 2.52-2.37(\mathrm{~m}, 2 \mathrm{H})$. ${ }^{13} \mathrm{C} \mathrm{NMR}\left(101 \mathrm{MHz}, \mathrm{CDCl}_{3}\right) \delta 198.1,172.9,142.6,142.5,136.2,132.1,129.8,129.2,129.2,128.7$, $128.7,128.3,127.6,127.5,51.8,48.3,47.5,44.2,40.3 . \mathrm{mp}: 169.9-171.3{ }^{\circ} \mathrm{C}$.

Methyl-6-oxo-3,4-diphenyl-6-(thiophen-2-yl)hexanoate (13): Synthesized from benzaldehyde, 2-acetylthiophene, cinnamaldehyde and methanol according to the representative procedure but purified by column chromatography (with petroleum ether $\left(40-60{ }^{\circ} \mathrm{C}\right) /$ EtOAc mixture, $25 \mathrm{~mL} / \mathrm{min}$, petroleum ether $100 \% \rightarrow 6 \% \rightarrow 8 \% \rightarrow 50 \%$ EtOAc in petroleum ether). Obtained as a white solid (103.3 mg, $0.27 \mathrm{mmol}, 83 \%$ yield, 20:1 dr). ${ }^{1} \mathrm{H}$ NMR (400 MHz, $\left.\mathrm{CDCl}_{3}\right) \delta 7.50-7.47(\mathrm{~m}, 1 \mathrm{H}), 7.42-7.37(\mathrm{~m}, 1 \mathrm{H})$, 7.36-7.26 (m, 8H), 7.25-7.15 (m, 2H), 6.99-6.95 (m, 1H), 3.63-3.53 (m, 1H), 3.47-3.39 (m, 1H), 3.37 (s, 3H), $3.17(\mathrm{dd}, J=16.1,10.4 \mathrm{~Hz}, 1 \mathrm{H}), 2.83(\mathrm{dd}, J=16.1,3.5 \mathrm{~Hz}, 1 \mathrm{H}), 2.52-2.36(\mathrm{~m}, 2 \mathrm{H}) .{ }^{13} \mathrm{C}$ NMR $\left(101 \mathrm{MHz}, \mathrm{CDCl}_{3}\right) \delta 191.4,172.6,144.5,142.2,142.1,133.5,131.7,128.9,128.8,128.4,128.4,127.9$, 127.3, 127.1, 51.5, 47.9, 47.3, 44.7, 40.0. IR (KBr) $v_{\max } / \mathrm{cm}^{-1}: 3940(\mathrm{br}, \mathrm{w}), 3082(\mathrm{w}), 3026(\mathrm{w}), 2916$ (w), 1730 (vs), 1654 (vs), 1418 (s), 1376 (w), 1240 (s), 1156 (s), 1072 (w), 782 (w), 702 (m), 556 (m). $\mathrm{mp}: 192.7-193.4^{\circ} \mathrm{C}$.

Methyl-6-(3,4-dimethoxyphenyl)-6-oxo-3,4-diphenylhexanoate (14): Synthesized from benzaldehyde, 3,4-dimethoxyacetophenone, cinnamaldehyde and methanol according to the representative procedure. Obtained as a white solid (208.3 mg, $0.48 \mathrm{mmol}, 68 \%$ yield, 20:1 dr). ${ }^{1} \mathrm{H}$ NMR (400 $\mathrm{MHz}, \mathrm{CDCl}_{3}$ ) $\delta$ 7.39-7.27 (m, 8H), 7.26-7.15 (m, 4H), 6.77-6.72 (m, 1H), $3.88(\mathrm{~s}, 3 \mathrm{H}), 3.81(\mathrm{~s}, 3 \mathrm{H}) .3 .63-3.54$ (m, 1H), 3.48-3.39 (m, 1H), $3.37(\mathrm{~s}, 3 \mathrm{H}), 3.22(\mathrm{dd}, J=16.4,10.7 \mathrm{~Hz}, 1 \mathrm{H}), 2.86(\mathrm{dd}, J=16.4,3.4 \mathrm{~Hz}$, 1H), 2.51-2.37 (m, 2H). ${ }^{13} \mathrm{C}$ NMR (101 MHz, $\left.\mathrm{CDCl}_{3}\right) \delta 197.3,172.7,153.1,148.9,142.5,142.4,130.4$, $128.8,128.8,128.5,128.3,127.2,127.0,122.6,110.2,109.9,56.1,56.0,51.5,48.0,47.3,43.4,40.1$. IR (KBr) $v_{\max } / \mathrm{cm}^{-1}: 3062(\mathrm{w}), 2940(\mathrm{~m}), 2846(\mathrm{w}), 1730$ (vs), 1666 (vs), 1590 (vs), 1514 (m), 1458 (s), 1420 (s), 1376 (m), 1346 (m), 1274 (vs), 1226 (vs), 1150 (vs), 1024 (s), 766 (m), 700 (s), 556 (m). $\mathrm{mp}: 147.0-149.0^{\circ} \mathrm{C}$.

Methyl-6-(naphthalen-2-yl)-6-oxo-3,4-diphenylhexanoate (15): Synthesized from benzaldehyde, $2^{\prime}$-acetonapthone, cinnamaldehyde and methanol according to the representative procedure. Obtained as a white solid (195.2 mg, $0.46 \mathrm{mmol}, 65 \%$ yield, 20:1 dr). ${ }^{1} \mathrm{H}$ NMR (400 MHz, $\left.\mathrm{CDCl}_{3}\right) \delta 8.09(\mathrm{~s}, 1 \mathrm{H})$, 
7.88-7.66 (m, 4H), 7.58-7.47 (m, 2H), 7.41-7.28 (m, 7H), 7.28-7.26 (m, 1H), 7.26-7.22 (m, 1H), 7.21-7.15 (m, 1H), 3.69-3.60 (m, 1H), 3.53-345 (m, 1H), 3.42-3.31 (m, 4H), 3.06 (dd, J = 16.2, 3.3 Hz, 1H), 2.54-2.38 (m, 2H). ${ }^{13} \mathrm{C}$ NMR (101 MHz, $\left.\mathrm{CDCl}_{3}\right) \delta 198.8,172.7,142.4,142.4,135.5,134.5,132.5$, 129.6, 129.6, 128.9, 128.8, 128.5, 128.4, 128.3, 127.8, 127.3, 127.1, 126.8, 123.9, 51.5, 48.0, 47.4, 44.1, 40.1. IR (KBr) $v_{\max } / \mathrm{cm}^{-1}: 3060(\mathrm{~m}), 3030(\mathrm{~m}), 2948(\mathrm{w}), 2846(\mathrm{w}), 1730(\mathrm{vs}), 1676$ (vs), $1498(\mathrm{~m})$, $1438(\mathrm{~m}), 1378$ (m), 1280 (m), 1232 (s), 1154 (s), 998 (w), 948 (w), 860 (w), 820 (w), 774 (m), 700 (s), $564(\mathrm{~m}), 474$ (m). mp: $198.7-200.0{ }^{\circ} \mathrm{C}$.

Methyl-6-oxo-3,6-diphenyl-4-(p-tolyl)hexanoate (16) [40]: Synthesized from p-tolualdehyde, acetophenone, cinnamaldehyde and methanol according to the representative procedure. Obtained as a white solid (204.6 mg, $0.53 \mathrm{mmol}, 78 \%$ yield, 20:1 dr). ${ }^{1} \mathrm{H}$ NMR (400 $\left.\mathrm{MHz}, \mathrm{CDCl}_{3}\right) \delta 7.65-7.60$ (m, 2H), 7.47-7.41 (m, 1H), 7.37-7.28 (m, 6H), 7.25-7.17 (m, 3H), 7.26-7.12 (m, 2H), 3.59-3.51 (m, 1H), 3.44-3.40 (m, 1H) 3.37 (s, 3H), $3.24(\mathrm{dd}, J=16.8,10.4 \mathrm{~Hz}, 1 \mathrm{H}), 2.88$ (dd, $J=16.8,3.3 \mathrm{~Hz}$, 1H), 2.50-2.38 (m, 2H) $2.28(\mathrm{~s}, 3 \mathrm{H}) .{ }^{13} \mathrm{C} \mathrm{NMR}\left(101 \mathrm{MHz}, \mathrm{CDCl}_{3}\right) \delta 198.2,172.8,142.4,139.4,137.2$, 136.6, 132.8, 129.8, 128.9, 128.5, 128.4, 128.3, 128.0, 127.2, 51.4, 48.1, 46.6, 44.0, 40.1, 21.2. $\mathrm{mp}: 156.7-157.9^{\circ} \mathrm{C}$.

Methyl-6-oxo-3,6-diphenyl-4-(m-tolyl)hexanoate (17) [40]: Synthesized from $m$-tolualdehyde, acetophenone, cinnamaldehyde and methanol according to the representative procedure. Obtained as a white solid (181.6 mg, $0.47 \mathrm{mmol}, 66 \%$ yield, 20:1 dr). ${ }^{1} \mathrm{H} \mathrm{NMR}\left(400 \mathrm{MHz}, \mathrm{CDCl}_{3}\right) \delta 7.66-7.59$ (m, 2H), 7.47-7.41 (m, 1H), 7.36-7.27 (m, 6H), 7.24-7.06 (m, 4H), $6.99(\mathrm{~m}, 1 \mathrm{H}), 3.59-3.51(\mathrm{~m}, 1 \mathrm{H})$, 3.44-3.38 (m, 1H), 3.37 (s, 3H), $3.26(\mathrm{dd}, J=16.7,10.1 \mathrm{~Hz}, 1 \mathrm{H}), 2.89$ (dd, $J=16.7,3.3 \mathrm{~Hz}, 1 \mathrm{H})$, 2.51-2.37 (m, 2H), 2.32 (s, 3H). $\left.{ }^{13} \mathrm{C} \mathrm{NMR} \mathrm{(101} \mathrm{MHz,} \mathrm{CDCl}_{3}\right) \delta 198.8,172.8,142.5,142.3,138.3,137.2$, 132.8, 129.2, 128.8, 128.6, 128.4, 128.4, 128.0, 127.8, 127.2, 125.4, 51.4, 48.0, 46.9, 43.9, 40.1, 21.7. $\mathrm{mp}: 172.2-173.0^{\circ} \mathrm{C}$.

Methyl-6-oxo-3,6-diphenyl-4-(o-tolyl)hexanoate (18) [40]: Synthesized from o-tolualdehyde, acetophenone, cinnamaldehyde and methanol according to the representative procedure. Obtained as a white solid (208.6 mg, $0.54 \mathrm{mmol}, 76 \%$ yield, 20:1 dr). ${ }^{1} \mathrm{H} \mathrm{NMR}\left(400 \mathrm{MHz}, \mathrm{CDCl}_{3}\right) \delta 7.65-7.59$ $(\mathrm{m}, 2 \mathrm{H}), 7.46-7.41(\mathrm{~m}, 1 \mathrm{H}), 7.37-7.27(\mathrm{~m}, 7 \mathrm{H}), 7.25-7.20(\mathrm{~m}, 1 \mathrm{H}), 7.14-7.11(\mathrm{~m}, 2 \mathrm{H}), 7.09-7.04$ (m, 1H), 3.97-3.87 (m, 1H), 3.44-3.28 (m, 5H), 2.91 (dd, J = 16.7, 3.5 Hz, 1H), 2.55-2.49 (m, 1H) 2.46 (s, 3H), $2.36(\mathrm{dd}, J=15.4,4.3 \mathrm{~Hz}, 1 \mathrm{H}) .{ }^{13} \mathrm{C} \mathrm{NMR}\left(101 \mathrm{MHz}, \mathrm{CDCl}_{3}\right) \delta 198.9,172.6,142.4,141.1,137.5$, 137.2, 132.8, 130.6, 128.9, 128.5, 128.4, 128.0, 127.2, 126.7, 126.6, 125.9, 51.5, 49.1, 44.1, 40.9, 39.1, 20.3. mp: $134.0-139.8^{\circ} \mathrm{C}$.

Methyl-4-(4-methoxyphenyl)-6-oxo-3,6-diphenylhexanoate (19) [40]: Synthesized from p-methoxybenzaldehyde, acetophenone, cinnamaldehyde and methanol according to the representative procedure. Obtained as a white solid $\left(205.9 \mathrm{mg}, 0.51 \mathrm{mmol}, 75 \%\right.$ yield, 20:1 dr). ${ }^{1} \mathrm{H} \mathrm{NMR}(400 \mathrm{MHz}$, $\left.\mathrm{CDCl}_{3}\right) \delta$ 7.65-7.60 (m, 2H), 7.47-7.42 (m, 1H), 7.36-7.28 (m, 6H), 7.25-7.19 (m, 3H), 6.85-6.79 $(\mathrm{m}, 2 \mathrm{H}), 3.76(\mathrm{~s}, 3 \mathrm{H}), 3.58-3.50(\mathrm{~m}, 1 \mathrm{H}), 3.43-3.34(\mathrm{~m}, 4 \mathrm{H}), 3.22(\mathrm{dd}, J=16.6,10.4 \mathrm{~Hz}, 1 \mathrm{H}), 2.87$ (dd, $J=16.6,3.4 \mathrm{~Hz}, 1 \mathrm{H}), 2.49-2.39(\mathrm{~m}, 2 \mathrm{H}) .{ }^{13} \mathrm{C} \mathrm{NMR}\left(101 \mathrm{MHz}, \mathrm{CDCl}_{3}\right) \delta 198.9,172.8,158.5,142.4$, $137.2,134.4,132.9,129.4,128.9,128.5,128.3$ 128.0, 127.2, 114.2, 55.3, 51.5, 48.2, 46.3, 44.0, 40.0. mp: $143.4-145.7^{\circ} \mathrm{C}$. 
Methyl-4-(2-methoxyphenyl)-6-oxo-3,6-diphenylhexanoate (20): Synthesized from $o$-methoxybenzaldehyde, acetophenone, cinnamaldehyde and methanol according to the representative procedure. Obtained as a white solid (198.1 mg, $0.49 \mathrm{mmol}, 70 \%$ yield, 20:1 dr). ${ }^{1} \mathrm{H} \mathrm{NMR}(400 \mathrm{MHz}$, $\left.\mathrm{CDCl}_{3}\right) \delta$ 7.64-7.58 (m, 2H), 7.46-7.40 (m, 1H), 7.38-7.28 (m, 6H), 7.25-7.13 (m, 3H), 6.92-6.80 $(\mathrm{m}, 2 \mathrm{H}), 4.00-3.92(\mathrm{~m}, 1 \mathrm{H}), 3.82(\mathrm{~s}, 3 \mathrm{H}), 3.63-3.52(\mathrm{~m}, 1 \mathrm{H}), 3.43-3.37(\mathrm{~m}, 1 \mathrm{H}), 3.35(\mathrm{~s}, 3 \mathrm{H}), 2.88$ $(\mathrm{dd}, J=16.6,3.4 \mathrm{~Hz}, 1 \mathrm{H}), 2.57-2.36(\mathrm{~m}, 2 \mathrm{H}) .{ }^{13} \mathrm{C} \mathrm{NMR}\left(101 \mathrm{MHz}, \mathrm{CDCl}_{3}\right) \delta 199.3,172.9,157.9,142.9$, 137.3 , 132.6, 130.2, 129.5, 128.7, 128.5, 128.3, 128.0, 127.1, 120.9, 111.1, 55.6, 51.3, 46.9, 42.4, 39.9. IR (KBr) $v_{\max } / \mathrm{cm}^{-1}: 3060(\mathrm{w}), 3026(\mathrm{w}), 2946$ (m), $1738(\mathrm{vs}), 1684(\mathrm{vs}), 1596(\mathrm{w}), 1494(\mathrm{~m}), 1454$ (m), 1378 (w), 1344 (w), 1242 (vs), 1156 (s), 1078 (w), 752 (s), 696 (s), 584 (w). mp: 110.8-112.4 ${ }^{\circ} \mathrm{C}$.

Methyl-4-(4-chlorophenyl)-6-oxo-3,6-diphenylhexanoate (21): Synthesized from p-chlorobenzaldehyde, acetophenone, cinnamaldehyde and methanol according to the representative procedure. Obtained as a white solid (304.1 mg, $0.75 \mathrm{mmol}, 88 \%$ yield, 20:1 dr). ${ }^{1} \mathrm{H}$ NMR (400 MHz, $\left.\mathrm{CDCl}_{3}\right) \delta$ 7.66-7.61 (m, 2H), 7.49-7.44 (m, 1H), 7.38-7.27 (m, 8H), 7.25-7.14 (m, 3H), 3.64-3.52 (m, 1H), 3.46-3.35 (m, 4H), $3.23(\mathrm{dd}, J=16.9,10.4 \mathrm{~Hz}, 1 \mathrm{H}) 2.9(\mathrm{dd}, J=17.0,3.4 \mathrm{~Hz}, 1 \mathrm{H}), 2.51-2.36$ (m, 2H). ${ }^{13} \mathrm{C} \mathrm{NMR}\left(101 \mathrm{MHz}, \mathrm{CDCl}_{3}\right) \delta 198.4,172.5,141.94,141.1,137.0,133.1,132.7,129.9,129.0$, 128.9, 128.6, 128.3, 128.0, 127.4, 51.5, 47.8, 46.3, 43.7, 39.9. IR (KBr) $v_{\max } / \mathrm{cm}^{-1}: 3060(\mathrm{w}), 3026(\mathrm{w})$, 2946 (w), 1734 (vs), 1676 (vs), 1494 (m), 1438 (m), 1380 (m), 1336 (w), 1276 (m), 1236 (s), 1166 (s), 1096 (w), 1002 (m), 832 (m), 754 (m), 696 (m), 554 (m). mp: 179.1-180.2 ${ }^{\circ} \mathrm{C}$.

Methyl-4-(4-bromophenyl)-6-oxo-3,6-diphenylhexanoate (22) [40]: Synthesized from p-bromobenzaldehyde, acetophenone, cinnamaldehyde and methanol according to the representative procedure. Obtained as a white solid $\left(242.0 \mathrm{mg}, 0.54 \mathrm{mmol}, 74 \%\right.$ yield, 20:1 dr). ${ }^{1} \mathrm{H} \mathrm{NMR}$ (400 MHz, $\left.\mathrm{CDCl}_{3}\right) \delta 7.66-7.62(\mathrm{~m}, 2 \mathrm{H}), 7.49-7.39(\mathrm{~m}, 3 \mathrm{H}), 7.36-7.28(\mathrm{~m}, 6 \mathrm{H}), 7.25-7.17(\mathrm{~m}, 3 \mathrm{H}), 3.63-3.52$ (m, 1H), 3.44-3.36 (m, 4H), $3.23(\mathrm{dd}, J=16.9,10.6 \mathrm{~Hz}, 1 \mathrm{H}) 2.9(\mathrm{dd}, J=16.9,3.1 \mathrm{~Hz}, 1 \mathrm{H}), 2.51-2.35$ $(\mathrm{m}, 2 \mathrm{H}){ }^{13} \mathrm{C}$ NMR $\left(101 \mathrm{MHz}, \mathrm{CDCl}_{3}\right) \delta 198.2,172.3,141.7,141.4,136.8,132.9,131.7,130.1,128.8$, $128.4,128.1,127.8,127.2,120.6,51.4,47.6,46.1,43.5,39.7 . \mathrm{mp}: 170.2-172.0{ }^{\circ} \mathrm{C}$.

Methyl-4-(furan-2-yl)-6-oxo-3,6-diphenylhexanoate (23) [40]: Synthesized form furfural, acetophenone, cinnamaldehyde and methanol according to the representative procedure. Obtained as a brown solid (135.8 mg, $0.37 \mathrm{mmol}, 52 \%$ yield, 20:1 dr). ${ }^{1} \mathrm{H}$ NMR (400 $\left.\mathrm{MHz}, \mathrm{CDCl}_{3}\right) \delta 7.74-7.69$ (m, 2H), 7.51-7.45 (m, 1H), 7.39-7.26 (m, 7H), 7.24-7.19 (m, 1H), 6.26-6.21 (m, 1H), 6.15-6.11 (m, 1H), 3.79-3.71 (m, 1H), 3.57-3.47 (m, $1 \mathrm{H}), 3.44(\mathrm{~s}, 3 \mathrm{H}), 3.42-3.34(\mathrm{~m}, 1 \mathrm{H}), 2.82(\mathrm{dd}, J=16.7,3.5 \mathrm{~Hz}, 1 \mathrm{H})$, 2.61-2.48 (m, 2H). ${ }^{13} \mathrm{C}$ NMR (101 MHz, $\left.\mathrm{CDCl}_{3}\right) \delta 198.4,172.4,155.0,141.9,141.7,137.0,133.0$, $128.9,128.5,128.2,128.1,127.3,110.3,107.9,51.6,46.2,41.1,40.4,39.7 . \mathrm{mp}: 142.7-143.4{ }^{\circ} \mathrm{C}$.

Methyl (E)-4-(2-oxo-2-phenylethyl)-3,6-diphenylhex-5-enoate (24): Synthesized from acetophenone, cinnamaldehyde and methanol according to the representative procedure. Obtained as a white solid (152.3 mg, $0.38 \mathrm{mmol}, 55 \%$ yield, $20: 1 \mathrm{dr}) .{ }^{1} \mathrm{H} \mathrm{NMR}\left(400 \mathrm{MHz}, \mathrm{CDCl}_{3}\right) \delta 7.66-7.61(\mathrm{~m}, 2 \mathrm{H})$, 7.47-7.41 (m, 1H), 7.36-7.26 (m, 10H), 7.25-7.15 (m, 2H), 3.65-3.56 (m, 1H), $6.46(\mathrm{~d}, J=15.8 \mathrm{~Hz}, 1 \mathrm{H})$, $6.04(\mathrm{dd}, J=15.8,9.3 \mathrm{~Hz}, 1 \mathrm{H}) 3.48-3.40(\mathrm{~m}, 1 \mathrm{H}) 3.37(\mathrm{~s}, 3 \mathrm{H}), 3.28(\mathrm{dd}, J=16.6,10.2 \mathrm{~Hz}, 1 \mathrm{H})$, 2.90-2.87 (m, 1H), 2.52-2.37 (m, 2H). $\left.{ }^{13} \mathrm{C} \mathrm{NMR} \mathrm{(101} \mathrm{MHz,} \mathrm{CDCl}_{3}\right)$ 8199.0, 172.9, 142.4, 137.3, 137.0, $132.9,131.0,128.9,128.6,128.3,128.1,127.5,127.2,126.4,77.2,51.6,46.4,45.6,42.5,40.2$. IR (KBr) 
$v_{\max } / \mathrm{cm}^{-1}$ : $3450(\mathrm{br}, \mathrm{w}), 3060(\mathrm{w}), 3026(\mathrm{w}), 2948(\mathrm{w}), 2890(\mathrm{w}), 1732(\mathrm{vs}), 1678(\mathrm{vs}), 1596(\mathrm{w}), 1494(\mathrm{w})$, 1440 (m), 1376 (m), 1272 (m), 1272 (s), 1166 (s), 968 (m), 752 (s), 696 (s). mp: 137.0-138.4 ${ }^{\circ} \mathrm{C}$.

Methyl-6-oxo-4,6-diphenyl-3-(p-tolyl)hexanoate (25) [40]: Synthesized from benzaldehyde, acetophenone, 4-methylcinnamaldehyde and methanol according to the representative procedure but purified by column chromatography (with petroleum ether $\left(40-60{ }^{\circ} \mathrm{C}\right) /$ EtOAc mixture, $25 \mathrm{~mL} / \mathrm{min}$, petroleum ether $100 \% \rightarrow 6 \% \rightarrow 8 \% \rightarrow 50 \%$ EtOAc in petroleum ether). Obtained as a white solid (112.1 mg, $0.29 \mathrm{mmol}, 84 \%$ yield, 20:1 dr). ). ${ }^{1} \mathrm{H}$ NMR (400 MHz, $\left.\mathrm{CDCl}_{3}\right) \delta$ 7.69-7.59 (m, 2H), 7.47-7.42 (m, 1H), 7.34-7.26 (m, 6H), 7.24-7.11 (m, 5H), 3.62-3.51 (m, 1H), 3.44-3.35 (m, 4H), 3.27 (dd, J=16.7, $10.5 \mathrm{~Hz}$, $1 \mathrm{H}), 2.92(\mathrm{dd}, J=16.7,3.5 \mathrm{~Hz}, 1 \mathrm{H}), 2.50-2.36(\mathrm{~m}, 2 \mathrm{H}), 2.31(\mathrm{~s}, 3 \mathrm{H}) .{ }^{13} \mathrm{C} \mathrm{NMR}\left(101 \mathrm{MHz}, \mathrm{CDCl}_{3}\right)$ $\delta 198.8,172.7,142.7,139.1,137.2,136.7,132.8,129.5,128.7,128.4,128.4,128.2,128.0,126.9,51.4$, 47.6, 47.0, 44.0, 40.1, 21.2. mp: $163.2-164.5^{\circ} \mathrm{C}$.

Methyl-3-(4-methoxyphenyl)-6-oxo-4,6-diphenylhexanoate (26) [40]: Synthesized from benzaldehyde, acetophenone, 4-methoxycinnamaldehyde and methanol according to the representative procedure. Obtained as a white solid $(199.7 \mathrm{mg}, 0.50 \mathrm{mmol}, 71 \%$ yield, $20: 1 \mathrm{dr}) .{ }^{1} \mathrm{H} \mathrm{NMR}(400 \mathrm{MHz}$, $\left.\mathrm{CDCl}_{3}\right) \delta$ 7.67-7.61 (m, 2H), 7.48-7.42 (m, 1H), 7.36-7.28 (m, 6H), 7.25-7.15 (m, 3H), 6.88-6.83 (m, 2H), $3.78(\mathrm{~s}, 3 \mathrm{H}), 3.60-3.52(\mathrm{~m}, 1 \mathrm{H}), 3.42-3.33(\mathrm{~m}, 4 \mathrm{H}), 3.25(\mathrm{dd}, J=16.5,10.0 \mathrm{~Hz}, 1 \mathrm{H}), 2.93$ $(\mathrm{dd}, J=16.5,3.4 \mathrm{~Hz}, 1 \mathrm{H}), 2.47-2.34(\mathrm{~m}, 2 \mathrm{H}) .{ }^{13} \mathrm{C} \mathrm{NMR}\left(101 \mathrm{MHz}, \mathrm{CDCl}_{3}\right) \delta 198.8,172.8,158.7,142.7$, 137.2, 134.1, 132.9, 129.3, 128.8, 128.4, 128.4, 128.0, 127.0, 114.2, 55.3, 51.5, 47.2, 47.2, 44.0, 40.2. mp: $160.8-161.7^{\circ} \mathrm{C}$.

Methyl-3-(4-chlorophenyl)-6-oxo-4,6-diphenylhexanoate (27) [40]: Synthesized from benzaldehyde, acetophenone, 4-chlorocinnamaldehyde and methanol according to the representative procedure. Obtained as a white solid (187.9 mg, $0.46 \mathrm{mmol}, 66 \%$ yield, 20:1 dr). ${ }^{1} \mathrm{H} \mathrm{NMR}(400 \mathrm{MHz}$, $\left.\mathrm{CDCl}_{3}\right) \delta$ 7.70-7.61 (m, 2H), 7.50-7.43 (m, 1H), 7.39-7.23 (m, 10H), 7.22-7.16 (m, 1H), 3.64-3.52 (m, 1H), 3.49-3.44 (m, 1H), $3.40(\mathrm{~s}, 3 \mathrm{H}), 3.28(\mathrm{dd}, J=16.8,9.6 \mathrm{~Hz}, 1 \mathrm{H}), 2.94-2.85(\mathrm{~m}, 1 \mathrm{H}), 2.50-2.36$ (m, 2H). ${ }^{3} \mathrm{C}$ NMR $\left(101 \mathrm{MHz}, \mathrm{CDCl}_{3}\right) \delta(\mathrm{s}), 172.5,142.2,140.8,137.1,133.0,129.7,129.0,128.9,128.5$, $128.4,127.9,127.1,51.6,47.3,46.7,43.7,39.8 . \mathrm{mp}: 180.5-181.3{ }^{\circ} \mathrm{C}$.

Methyl-3-(4-fluorophenyl)-6-oxo-4,6-diphenylhexanoate (28): Synthesized from benzaldehyde, acetophenone, 4-fluorocinnamaldehyde and methanol according to the representative procedure but purified by column chromatography (with petroleum ether $\left(40-60{ }^{\circ} \mathrm{C}\right) / \mathrm{EtOAc}$ mixture, $25 \mathrm{~mL} / \mathrm{min}$, petroleum ether $100 \% \rightarrow 6 \% \rightarrow 8 \% \rightarrow 50 \%$ EtOAc in petroleum ether). Obtained as a white solid (91.8 mg, $0.24 \mathrm{mmol}, 72 \%$ yield, 20:1 dr). ${ }^{1} \mathrm{H}$ NMR (400 MHz, $\left.\mathrm{CDCl}_{3}\right) \delta$ 7.67-7.61 (m, 2H), 7.49-7.43 (m, 1H), 7.36-7.26 (m, 8H), 7.22-7.14 (m, 1H), 7.04-6.94 (m, 2H), 3.63-3.54 (m, 1H), 3.52-3.40 (m, 1H), 3.39 $(\mathrm{s}, 3 \mathrm{H}), 3.32-3.22(\mathrm{~m}, 1 \mathrm{H}), 2.95-2.85(\mathrm{~m}, 1 \mathrm{H}), 2.49-2.37(\mathrm{~m}, 2 \mathrm{H}) .{ }^{13} \mathrm{C} \mathrm{NMR}\left(101 \mathrm{MHz}, \mathrm{CDCl}_{3}\right) \delta 198.5$, 172.5, 163.2, 160.7, 142.4, 137.9, 137.1, 133.0, 129.9, 129.8, 128.5, 128.4, 127.9, 127.1, 115.8, 115.6, 51.5, 47.2, 46.9, 43.8, 40.0. ${ }^{19} \mathrm{~F}$ NMR $\left(376 \mathrm{MHz}, \mathrm{CDCl}_{3}\right)$-115.6. IR (KBr) $v_{\max } / \mathrm{cm}^{-1}: 3030(\mathrm{w}), 2950$ (w), 2888 (w), 1728 (vs), 1682 (vs), 1600 (w), 1508 (m), 1444 (m), 1374 (m), 1234 (vs), 1158 (s), 1006 (w), $834(\mathrm{~m}), 738(\mathrm{~m}), 698(\mathrm{~m}), 560(\mathrm{~m}) . \mathrm{mp}: 169.4-174.6^{\circ} \mathrm{C}$. 
Ethyl-6-oxo-3,4,6-triphenylhexanoate (29) [40]: Synthesized from benzaldehyde, acetophenone, cinnamaldehyde and ethanol according to the representative procedure. Obtained as a white solid (193.6 mg, $0.50 \mathrm{mmol}, 73 \%$ yield, 20:1 dr). ${ }^{1} \mathrm{H}$ NMR (400 MHz, $\left.\mathrm{CDCl}_{3}\right) \delta$ 7.66-7.60 (m, 2H), 7.47-7.41 (m, 1H), 7.39-7.27 (m, 9H), 7.25-7.13 (m, 3H), $3.82(\mathrm{q}, J=7.0 \mathrm{~Hz}, 2 \mathrm{H}), 3.64-3.56(\mathrm{~m}, 1 \mathrm{H})$, $3.47-3.36(\mathrm{~m}, 1 \mathrm{H}), 3.28(\mathrm{dd}, J=16.7,10.2 \mathrm{~Hz}, 1 \mathrm{H}), 2.91(\mathrm{dd}, J=16.7,3.2 \mathrm{~Hz}, 1 \mathrm{H}), 2.50-2.34(\mathrm{~m}, 2 \mathrm{H})$, $0.97(\mathrm{t}, J=7.0 \mathrm{~Hz}, 3 \mathrm{H}) .{ }^{13} \mathrm{C} \mathrm{NMR}\left(101 \mathrm{MHz}, \mathrm{CDCl}_{3}\right) \delta 198.7,172.3,142.5,142.2,137.2,132.9,128.8$, $128.8,128.5,128.0,127.2,127.0,60.2,48.1,47.1,43.9,40.3,14.1 . \mathrm{mp}: 172.4-173.4{ }^{\circ} \mathrm{C}$.

Benzyl-6-oxo-3,4,6-triphenylhexanoate (30) [40]: Synthesized from benzaldehyde, acetophenone, cinnamaldehyde and benzyl alcohol according to the representative procedure. Obtained as a white solid (180.2 mg, $0.40 \mathrm{mmol}$, 59\% yield, 20:1 dr). ${ }^{1} \mathrm{H}$ NMR (400 MHz, $\left.\mathrm{CDCl}_{3}\right) \delta 7.66-7.60(\mathrm{~m}, 2 \mathrm{H})$, 7.49-7.42 (m, 1H), 7.38-7.27 (m, 10H), 7.25-7.13 (m, 5H), 7.08-7.00 (m, 2H) 4.83 (m, 2H), 3.65-3.55 (m, 1H), 3.49-3.41 (m, 1H), 3.28 (dd, $J=16.6,10.2 \mathrm{~Hz}, 1 \mathrm{H}), 2.90(\mathrm{dd}, J=16.6,3.4 \mathrm{~Hz}, 1 \mathrm{H}), 2.57-2.42$ $(\mathrm{m}, 2 \mathrm{H}) .{ }^{13} \mathrm{C} \mathrm{NMR}\left(101 \mathrm{MHz}, \mathrm{CDCl}_{3}\right) \delta 198.7,172.2,142.5,142.1,137.2,135.9,132.9,128.9,128.8$, $128.5,128.5,128.1,128.0,127.3,127.0,66.1,48.1,47.1,43.9,40.2 . \mathrm{mp}: 178.0-179.2{ }^{\circ} \mathrm{C}$.

\section{Conclusions}

Herein, the first IL-mediated four-component synthesis of OTHOs is presented. The IL serves as a precatalyst that, upon deprotonation, generates the active NHC species. Careful base selection and usage of an IL-based precatalyst proved important for the success of the reaction, with excellent yields per bond-forming step (up to $>95 \%$ ). This one-pot, four-component OTHO synthesis is green in several perspectives: firstly, avoiding the pre-formation and isolation of chalcone saves time and resources. Secondly, a more environmentally friendly solvent is used compared to the previously reported protocol. Thirdly, the IL can be recycled and the reaction can be run up to five times without any negative effect on either yield or selectivity. The combination of a commercially available, inexpensive IL, with a large catalogue of readily available starting materials, makes the one-pot four-component assembly of OTHOs an ideal platform for the synthesis of diverse libraries of functionalized products, when run in a combinatorial fashion.

\section{Acknowledgments}

We gratefully acknowledge the Swedish Research Council VR and Formas, Magnus Bergvalls stiftelse and Wilhelm och Martina Lundgrens forskningsstiftelse for funding.

\section{Author Contributions}

A.A, L.T and H.S conceived and designed the experiments. A.A and L.T performed the experiments and analyzed the data. A.A, L.T and H.S wrote the paper.

\section{Conflicts of Interest}

The authors declare no conflict of interest. 


\section{References}

1. Zhang, Q.; Zhang, S.; Deng, Y. Recent advances in ionic liquid catalysis. Green. Chem. 2011, 13, 2619-2637.

2. Closson, A.; Johansson, M.; Backvall, J.-E. Ionic liquid-immobilized catalytic system for biomimetic dihydroxylation of olefins. Chem. Commun. 2004, 7, 1494-1495.

3. Luo, S.; Mi, X.; Zhang, L.; Liu, S.; Xu, H.; Cheng, J.-P. Functionalized chiral ionic liquids as highly efficient asymmetric organocatalysts for michael addition to nitroolefins. Angew. Chem. Int. Ed. 2006, 45, 3093-3097.

4. Luo, S.; Zhang, L.; Cheng, J.-P. Functionalized chiral ionic liquids: A new type of asymmetric organocatalysts and nonclassical chiral ligands. Chem. Asian J. 2009, 4, 1184-1195.

5. Siyutkin, D.E.; Kucherenko, A.S.; Zlotin, S.G. Ionic Liquid Organocatalysts; Wiley-VCH Verlag GmbH \& Co. KGaA: Weinheim, Germany, 2013; pp. 617-650.

6. Zalewska, K.; Branco, L.C. Organocatalysis with Chiral Ionic Liquids. Mini-Rev. Org. Chem. 2014, $11,141-153$.

7. Welton, T. Ionic liquids in catalysis. Coord. Chem. Rev. 2004, 248, 2459-2477.

8. Welton, T. Room-temperature ionic liquids. Solvents for synthesis and catalysis. Chem. Rev. 1999, 99, 2071-2084.

9. Martins, M.A.P.; Frizzo, C.P.; Moreira, D.N.; Zanatta, N.; Bonacorso, H.G. Ionic liquids in heterocyclic synthesis. Chem. Rev. 2008, 108, 2015-2050.

10. Aggarwal, A.; Lancaster, N.L.; Sethi, A.R.; Welton, T. The role of hydrogen bonding in controlling the selectivity of Diels-Alder reactions in room-temperature ionic liquids. Green Chem. 2002, 4, $517-520$.

11. Gholap, A.R.; Venkatesan, K.; Daniel, T.; Lahoti, R.J.; Srinivasan, K.V. Ultrasound promoted acetylation of alcohols in room temperature ionic liquid under ambient conditions. Green Chem. 2003, 5, 693-696.

12. Forsyth, S.A.; MacFarlane, D.R.; Thomson, R.J.; von Itzstein, M. Rapid, clean, and mild O-acetylation of alcohols and carbohydrates in an ionic liquid. Chem. Commun. 2002, 7, 714-715.

13. Dubreuil, J.F.; Bazureau, J.P. Rate accelerations of 1,3-dipolar cycloaddition reactions in ionic liquids. Tetrahedron Lett. 2000, 41, 7351-7355.

14. Enders, D.; Balensiefer, T. Nucleophilic Carbenes in Asymmetric Organocatalysis. Acc. Chem. Res. 2004, 37, 534-541.

15. Enders, D.; Niemeier, O.; Henseler, A. Organocatalysis by N-heterocyclic carbenes. Chem. Rev. 2007, 107, 5606-5655.

16. List, B. Enamine catalysis is a powerful strategy for the catalytic generation and use of carbanion equivalents. Acc. Chem. Res. 2004, 37, 548-557.

17. Nair, V.; Bindu, S.; Sreekumar, V. N-Heterocyclic carbenes: Reagents, not just ligands! Angew. Chem. Int. Ed. 2004, 43, 5130-5135.

18. Marion, N.; Díez-González, S.; Nolan, S.P. N-heterocyclic carbenes as organocatalysts. Angew. Chem. Int. Ed. 2007, 46, 2988-3000.

19. Alba, A.-N.; Companyo, X.; Viciano, M.; Rios, R. Organocatalytic domino reactions. Curr. Org. Chem. 2009, 13, 1432-1474. 
20. Biju, A.T.; Kuhl, N.; Glorius, F. Extending NHC-catalysis: Coupling aldehydes with unconventional reaction partners. Acc. Chem. Res. 2011, 44, 1182-1195.

21. Bugaut, X.; Glorius, F. Organocatalytic umpolung: $N$-heterocyclic carbenes and beyond. Chem. Soc. Rev. 2012, 41, 3511-3522.

22. Davis, J.H., Jr.; Forrester, K.J. Thiazolium-ion based organic ionic liquids (OILs).1,2 Novel OILs which promote the benzoin condensation. Tetrahedron Lett. 1999, 40, 1621-1622.

23. Xu, L.-W.; Gao, Y.; Yin, J.-J.; Li, L.; Xia, C.-G. Efficient and mild benzoin condensation reaction catalyzed by simple 1-N-alkyl-3-methylimidazolium salts. Tetrahedron Lett. 2005, 46, 5317-5320.

24. Jiang, F.S.; Yu, H.; Gao, G.; Xie, R.G. Benzoin condensation in imidazolium based room-temperature ionic liquids. Chin. Chem. Lett. 2005, 16, 321-324.

25. Estager, J.; Lévêque, J.-M.; Turgis, R.; Draye, M. Solventless and swift benzoin condensation catalyzed by 1-alkyl-3-methylimidazolium ionic liquids under microwave irradiation. J. Mol. Catal. A 2006, 256, 261-264.

26. Estager, J.; Lévêque, J.-M.; Turgis, R.; Draye, M. Neat benzoin condensation in recyclable room-temperature ionic liquids under ultrasonic activation. Tetrahedron Lett. 2007, 48, 755-759.

27. Orsini, M.; Chiarotto, I.; Elinson, M.N.; Sotgiu, G.; Inesi, A. Benzoin condensation in 1,3-dialkylimidazolium ionic liquids via electrochemical generation of $N$-heterocyclic carbene. Electrochem. Commun. 2009, 11, 1013-1017.

28. Kelemen, Z.; Holloczki, O.; Nagy, J.; Nyulaszi, L. An organocatalytic ionic liquid. Org. Biomol. Chem. 2011, 9, 5362-5364.

29. Yu, F.-L.; Zhang, R.-L.; Xie, C.-X.; Yu, S.-T. Synthesis of thermoregulated phase-separable triazolium ionic liquids catalysts and application for Stetter reaction. Tetrahedron 2010, 66, 9145-9150.

30. Aupoix, A.; Vo-Thanh, G. Solvent-free synthesis of alkylthiazolium-based ionic liquids and their use as catalysts in the intramolecular Stetter reaction. Synlett 2009, 12, 1915-1920.

31. Yu, F.-L.; Jiang, J.-J.; Zhao, D.-M.; Xie, C.-X.; Yu, S.-T. Imidazolium chiral ionic liquid derived carbene-catalyzed conjugate umpolung for synthesis of $\gamma$-butyrolactones. RSC Adv. 2013, 3, 3996-4000.

32. Liu, D.; Zhang, Y.; Chen, E.Y.X. Organocatalytic upgrading of the key biorefining building block by a catalytic ionic liquid and N-heterocyclic carbenes. Green Chem. 2012, 14, 2738-2746.

33. Feroci, M.; Chiarotto, I.; Orsini, M.; Pelagalli, R.; Inesi, A. Umpolung reactions in an ionic liquid catalyzed by electrogenerated $N$-heterocyclic carbenes. Synthesis of saturated esters from activated $\alpha, \beta$-unsaturated aldehydes. Chem. Commun. 2012, 48, 5361-5363.

34. Forte, G.; Chiarotto, I.; Inesi, A.; Loreto, M.A.; Feroci, M. Electrogenerated N-heterocyclic carbene in ionic liquid: An insight into the mechanism of the oxidative esterification of aromatic aldehydes. Adv. Synth. Catal. 2014, doi:10.1002/adsc.201400163.

35. Chiarotto, I.; Feroci, M.; Sotgiu, G.; Inesi, A. Electrogenerated $N$-heterocyclic carbenes in the room temperature parent ionic liquid as an efficient medium for transesterification/acylation reactions. Eur. J. Org. Chem. 2013, 2013, 326-331.

36. Constable, D.J.C.; Jimenez-Gonzalez, C.; Henderson, R.K. Perspective on solvent use in the pharmaceutical industry. Org. Process Res. Dev. 2007, 11, 133-137. 
37. Jiménez-González, C.; Curzons, A.; Constable, D.C.; Cunningham, V. Expanding GSK's solvent selection guide-Application of life cycle assessment to enhance solvent selections. Clean Technol. Environ. Policy 2004, 7, 42-50.

38. Dunn, P.J. The importance of green chemistry in process research and development. Chem. Soc. Rev. 2012, 41, 1452-1461.

39. Dömling, A.; Ugi, I. Multicomponent reactions with isocyanides. Angew. Chem. Int. Ed. 2000, 39, 3168-3210.

40. Ta, L.; Axelsson, A.; Bijl, J.; Haukka, M.; Sundén, H. Ionic liquids as precatalysts in the highly stereoselective conjugate addition of $\alpha, \beta$-unsaturated aldehydes to chalcones. Chem. Eur. J. 2014, 20, 13889-13893.

41. Sundén, H.; Ta, L.; Axelsson, A. Highly stereoselective synthesis of 1,6-ketoesters mediated by ionic liquids: A three-component reaction enabling rapid access to a new class of low molecular weight gelators. J. Vis. Exp. 2015, e53213, doi:10.3791/53213.

42. Holloczki, O.; Gerhard, D.; Massone, K.; Szarvas, L.; Nemeth, B.; Veszpremi, T.; Nyulaszi, L. Carbenes in ionic liquids. New J. Chem. 2010, 34, 3004-3009.

43. Guo, C.; Fleige, M.; Janssen-Müller, D.; Daniliuc, C.G.; Glorius, F. Switchable selectivity in an NHC-catalysed dearomatizing annulation reaction. Nat. Chem. 2015, 7, 842-847.

44. Feroci, M.; Chiarotto, I.; D’Anna, F.; Forte, G.; Noto, R.; Inesi, A. Stability and organocatalytic efficiency of N-heterocyclic carbenes electrogenerated in organic solvents from imidazolium ionic liquids. Electrochim. Acta 2015, 153, 122-129.

45. Wendler, F.; Todi, L.-N.; Meister, F. Thermostability of imidazolium ionic liquids as direct solvents for cellulose. Thermochim. Acta 2012, 528, 76-84.

(C) 2015 by the authors; licensee MDPI, Basel, Switzerland. This article is an open access article distributed under the terms and conditions of the Creative Commons Attribution license (http://creativecommons.org/licenses/by/4.0/). 